Opinion

\title{
Are Nociplastic Pain and Neuropathic Pain Different Pains?
}

\section{Katsuhiro Toda}

How to cite this paper: Toda, K. (2022). Are Nociplastic Pain and Neuropathic Pain Different Pains?. Universal Journal of Neuroscience, 1(1), 1-2. Retrieved from https://www.scipublications.com/journal/index.php/ujn/article/view/142

Received: September 17, 2021 Accepted: January 13, 2021 Published: January 14, 2022

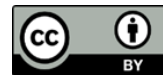

Copyright: (c) 2022 by the authors. Submitted for possible open access publication under the terms and conditions of the Creative Commons Attribution (CC BY) license (http://creativecommons.org/licenses /by/4.0/).
Department of Orthopedic Surgery, Hiroshima Clinic. 4th floor Fuji Ground, 2-1, Takara-machi, Naka-ku, Hiroshima, 730-0044, Japan

*Correspondence: goutattack@yahoo.co.jp

\begin{abstract}
The International Association for the Study of Pain has classified pain into nociceptive pain, neuropathic pain, and nociplastic pain based on the cause of the pain. At present, nociplastic pain is pain that is not nociceptive pain and has the following characteristics: no clear evidence of actual or threatened tissue damage causing the activation of peripheral nociceptors or evidence for disease or lesion of the somatosensory system causing the pain. If there is tissue damage, disease or lesion, it is neuropathic pain; if there is none, it is nociplastic pain. In other words, the difference in diagnosis is whether or not tissue damage, disease, or lesion can be found the current medical level (testing equipment). The treatment of nociplastic pain is almost the same as the treatment of neuropathic pain. Fibromyalgia is included in nociplastic pain. To my knowledge, of the nociplastic pain and neuropathic pain, fibromyalgia is the disease with the highest number of pharmacological and non-pharmacological treatments with evidence of efficacy. Effective treatments for fibromyalgia are often given to neuropathic pain. This expands treatment options. I presume that tissue damage, disease or lesion will be found in fibromyalgia through advances in the medical science by the time humans emigrate to Mars. The distinction between nociplastic pain and neuropathic pain complicates the diagnosis of chronic pain. However, the distinction does not improve the treatment outcomes. Medical science is the discipline to find a treatment method that can produce better outcomes. In the event of a medical controversy, the medical theory with better treatment outcomes should be adopted. It is desirable to combine nociplastic pain and neuropathic pain into one pain. This will simplify diagnosis and increase treatment options (improve treatment outcomes) in nociplastic pain and neuropathic pain.
\end{abstract}

Keywords: Nociceptive Pain; Neuropathic Pain; Fibromyalgia; Classification

The International Association for the Study of Pain has classified pain into nociceptive pain, neuropathic pain, and nociplastic pain based on the cause of the pain [1]. Nociceptive pain and neuropathic pain need to be distinguished because their treatments are completely different. When the two pains are combined, each treatment will be used in combination. However, the treatment of nociplastic pain is almost the same as the treatment of neuropathic pain. To my knowledge, of the nociplastic pain and neuropathic pain, fibromyalgia is the disease with the highest number of pharmacological and non-pharmacological treatments with evidence of efficacy. Fibromyalgia is included in nociplastic pain. Diabetic neuropathic pain and postherpetic neuralgia are representative diseases of neuropathic pain. They have less treatments with evidence of efficacy compared to fibromyalgia. Therefore, effective treatments for fibromyalgia are often given to them. This expands treatment options. We do not have to distinguish between nociplastic pain and neuropathic pain from the standpoint of the treatment.

At present, nociplastic pain is pain that is not nociceptive pain and has the following characteristics: no clear evidence of actual or threatened tissue damage causing the activation of peripheral nociceptors or evidence for disease or lesion of the somatosensory system causing the pain [1]. If there is tissue damage, disease or lesion, it is neuropathic pain; if there is none, it is nociplastic pain. In other words, the difference in diagnosis is 
whether or not tissue damage, disease, or lesion can be found at the current medical level (testing equipment). In Parkinson's disease, tissue damage, disease or lesion was not found when Cro-Magnon person were extinct, however, they are found through advances in the medical science. At present, in fibromyalgia, a typical disease of nociplastic pain, abnormalities in the brain have been found in research-level tests [2]. I presume that tissue damage, disease or lesion will be found in fibromyalgia through advances in the medical science by the time humans emigrate to Mars.

The prevalence of fibromyalgia, a typical disease of nociplastic pain, is only approximately $2 \%$ [3]. However, the combined prevalence of chronic widespread pain and chronic regional pain, which are presumed to be incomplete form or pre-stage of fibromyalgia, is at least $20 \%$ [3]. Because of the tremendous prevalence of fibromyalgia including the incomplete form, I believe that fibromyalgia is the most typical disease of chronic pain or neuropathic pain.

The distinction between nociplastic pain and neuropathic pain complicates the diagnosis of chronic pain. However, the distinction does not improve the treatment outcomes. Medical science is the discipline to find a treatment method that can produce better outcomes. In the event of a medical controversy, the medical theory with better treatment outcomes should be adopted. It is desirable to combine nociplastic pain and neuropathic pain into one pain. This will simplify diagnosis and increase treatment options (improve treatment outcomes) in nociplastic pain and neuropathic pain. Are nociplastic pain and neuropathic pain different pains? What is the purpose to distinguish between nociplastic pain and neuropathic pain?

Funding: No funding.

Conflicts of interest: My daughter is an employee of Nippon Zoki Pharmaceutical Co., Ltd.

Ethics approval: Not applicable.

\section{References}

[1] International Association for the Study of Pain. IASP Terminology. https://www.iasp-pain.org/Education/Content.aspx?ItemNumber=1698\#Nociplasticpain (accessed Sep.17, 2021)

[2] van Ettinger-Veenstra, H.; Boehme, R.; Ghafouri, B.; Olausson, H.; Wicksell, R.K.; Gerdle, B. Exploration of Functional Connectivity Changes Previously Reported in Fibromyalgia and Their Relation to Psychological Distress and Pain Measures. J. Clin. Med. 2020;9:3560.

[3] Toda, K.; Harada, T.; Prevalence, classification, and etiology of pain in Parkinson's disease: association between Parkinson's disease and fibromyalgia or chronic widespread pain. Tohoku. J. Ex.p Med. 2010;222:1-5. 